\title{
Resistance training-induced gains in muscle strength, body composition, and functional capacity are attenuated in elderly women with sarcopenic obesity
}

This article was published in the following Dove Press journal:

Clinical Interventions in Aging

\author{
Alessandro de Oliveira \\ Silva ${ }^{1,2}$ \\ Maurílio Tiradentes Dutra ${ }^{3}$ \\ Wilson Max Almeida \\ Monteiro de Moraes ${ }^{4}$ \\ Silvana Schwerz Funghetto ${ }^{3}$ \\ Darlan Lopes de Farias' \\ Paulo Henrique Fernandes \\ dos Santos ${ }^{3}$ \\ Denis Cesar Leite Vieira ${ }^{5}$ \\ Dahan da Cunha \\ Nascimento ${ }^{4,5}$ \\ Vânia Silva Macedo Orsano 4 \\ Brad J Schoenfeld ${ }^{6}$ \\ Jonato Prestes 4 \\ 'University Center of Brasilia \\ (UniCEUB), Brasília, Brazil; ${ }^{2}$ Integrated \\ Colleges of the Central Plateau \\ Educational Union (FACIPLAC), \\ Brasília, Brazil; ${ }^{3}$ University of Brasilia \\ (UnB), Brasília, Brazil; ${ }^{4}$ Catholic \\ University of Brasilia (UCB), Brasília, \\ Brazil; ${ }^{5}$ University Center of the \\ Federal District (UDF), Brasília, Brazil; \\ ${ }^{6}$ Department of Health Sciences, \\ CUNY Lehman College, Bronx, \\ NY, United States
}

\begin{abstract}
Objectives: The purpose of this study was to compare the effects of resistance training (RT) on body composition, muscle strength, and functional capacity in elderly women with and without sarcopenic obesity (SO).

Methods: A total of 49 women (aged $\geq 60$ years) were divided in two groups: without SO (non-SO, $\mathrm{n}=41$ ) and with $\mathrm{SO}(\mathrm{n}=8)$. Both groups performed a periodized RT program consisting of two weekly sessions for 16 weeks. All measures were assessed at baseline and postintervention, including anthropometry and body composition (dual-energy X-ray absorptiometry), muscle strength (one repetition maximum) for chest press and $45^{\circ}$ leg press, and functional capacity (stand up, elbow flexion, timed "up and go").

Results: After the intervention, only the non-SO group presented significant reductions in percentage body fat $(-2.2 \% ; P=0.006)$, waist circumference $(-2.7 \% ; P=0.01)$, waist-to-hip ratio $(-2.3 ; P=0.02)$, and neck circumference $(-1.8 \% ; P=0.03)$ as compared with baseline. Muscle strength in the chest press and biceps curl increased in non-SO only $(12.9 \%$ and $11.3 \%$, respectively), while $45^{\circ}$ leg press strength increased in non-SO (50.3\%) and SO (40.5\%) as compared with baseline. Performance in the chair stand up and timed "up and go" improved in non-SO only ( $21.4 \%$ and $-8.4 \%$, respectively), whereas elbow flexion performance increased in non-SO (23.8\%) and SO (21.4\%). Effect sizes for motor tests were of higher magnitude in the non-SO group, and in general, considered "moderate" compared to "trivial" in the SO group. Conclusion: Results suggest that adaptations induced by 16 weeks of RT are attenuated in elderly woman with SO, compromising improvements in adiposity indices and gains in muscle strength and functional capacity.
\end{abstract}

Keywords: aging, obesity, resistance training, sarcopenia

\section{Introduction}

The rapidly growing elderly population in most developed countries has resulted in an epidemiological confluence of risk factors for health-related conditions. In this context, the prevalence of obesity in older adults has doubled since 1980, and it continues to increase worldwide ${ }^{1}$ with more than a third of persons $\geq 65$ years of age in the USA considered obese. ${ }^{2}$ In addition, aging is accompanied by a progressive loss of muscle mass and poor physical function referred to as sarcopenia, which dramatically affects health status and quality of life in those afflicted. ${ }^{3}$ Coexistence of sarcopenia and obesity, termed sarcopenic obesity (SO), ${ }^{4}$ may act synergistically to exacerbate health-threatening effects.
Correspondence: Jonato Prestes Catholic University of Brasília (UCB), QS 07, Lote OI, EPTC - Bloco G, Taguatinga, Federal District, Brasilia 71966-700, Brazil Email jonatop@gmail.com (c)
hereby accept the Terms. Non-commercial uses of the work are permitted without any further permission from Dove Medical Press Limited, provided the work is properly attributed. For permission hereby accept the Terms. Non-commercial uses of the work are permitted without any further permission from Dove Mediect
for commercial use of this work, please see paragraphs 4.2 and 5 of our Terms (https://www.dovepress.com/terms.php). 
SO has been related to longer periods of hospitalization, ${ }^{5}$ higher health care costs, ${ }^{6}$ increased risk of cardiovascular diseases, ${ }^{7}$ and greater mortality ${ }^{8}$ than sarcopenia or obesity alone. Furthermore, elderly individuals with SO showed a higher risk for incurring functional limitations, notably in women. ${ }^{9,10}$ Women with SO have an odds ratio for two or more self-reported physical disabilities on the instrumental activities of daily living of 11.98 compared with 2.96 for sarcopenia and 2.15 for obesity alone. ${ }^{10}$ These negative outcomes suggest that sarcopenia and obesity have independent and additive adverse effects on health and that the efforts taken to promote healthy aging must consider both preventing obesity and maintaining or increasing skeletal muscle mass and function.

Despite significant and rising public health concerns, SO is a modifiable condition and can be treated with effective therapy. In this sense, it has been argued that exercise training interventions and, in particular, resistance training (RT) could ameliorate outcomes related to SO by promoting beneficial changes in muscle strength ${ }^{11,12}$ and adiposity indices. ${ }^{13}$ However, a more careful analysis of intervention studies in elderly individuals with SO has shown conflicting results with respect to improvements in physical function. Although some studies have reported increases in muscular strength and physical performance associated with regular RT in those with $\mathrm{SO},{ }^{12-14}$ others have failed to document improvements. ${ }^{11}$ Moreover, most studies to date have focused on older people with obesity and frailty ${ }^{15-17}$ or postmenopausal women separately ${ }^{18}$ and not with the SO condition. In addition, some studies used a combination of different modalities of exercises that make it difficult to evaluate the isolated effects of RT. Thus, the effects of RT in those with $\mathrm{SO}$ are not completely elucidated.

Obesity and aging are characterized by several abnormalities in skeletal muscle and, in particular, intramuscular lipid accumulation, which has been associated with low force generation, reduced regenerative capacity from injuries, resistance to anabolic stimulus (growth factors, hormones, amino acids, and exercise), ${ }^{19,20}$ and heightened local inflammatory pathways. ${ }^{21}$ All these abnormalities may impair exercise adaptations, ${ }^{22,23}$ potentially mitigating gains in muscle function.

Based on these assumptions, it is reasonable to hypothesize that elderly women with SO are less responsive to the benefits induced by RT as compared with elderly women without SO. Thus, the present study was undertaken to compare the effects of 16 weeks of RT on body composition, muscle strength, and functional capacity in elderly women with and without SO.

\section{Materials and methods Subjects}

Initially, 66 sedentary obese elderly women were recruited to participate in this study. Before participating in the experimental protocols, each subject underwent a complete physical examination that included clinical history, anthropometry, resting and exercise electrocardiogram, as well as orthopedic and blood pressure assessment. The following inclusion criteria were adopted: sedentary women, aged $\geq 60$ years, with a body fat percentage $>32 \%$. Obesity was determined as recommended by the National Institute of Diabetes and Digestive and Kidney Diseases, assuming a cutoff point of $30 \%$ for women. ${ }^{24}$ Exclusion criteria were as follows: smoking, sarcopenia alone, alcohol and drug abuse in the last 2 years, physical disability, diagnosis of diabetes, cardiovascular disease, hypertension, inflammatory and rheumatic diseases, autoimmune diseases, or the use of medications (such as beta blockers, hormone replacement therapy, anti-inflammatory, insulin). Enrollment was voluntary and written informed consent was obtained from each participant. The procedures complied with the Helsinki Declaration, and the experimental protocol was approved by the Catholic University of Brasília Ethics Committee (protocol no. 235/2010).

After screening for eligibility, 49 elderly women were selected to participate. Subjects were divided into two groups: those without $\mathrm{SO}$ (non-SO, $\mathrm{n}=41,66.0 \pm 4.0$ years, $63.7 \pm 10.78 \mathrm{~kg}, 151.2 \pm 6.2 \mathrm{~cm}$ ) and those with $\mathrm{SO}(\mathrm{SO}, \mathrm{n}=8$, $66.9 \pm 3.3$ years, $69.2 \pm 12.3 \mathrm{~kg}, 156.3 \pm 3.2 \mathrm{~cm}$ ). Both groups completed 16 weeks of regimented RT.

\section{Anthropometry and body composition}

Height (to the nearest $0.1 \mathrm{~cm}$ ) and body mass (to the nearest $0.1 \mathrm{~kg}$ ) were measured and utilized to determine body mass index (BMI, body mass $/$ height $^{2}$ ). All circumferences were obtained in triplicate using a nonelastic tape measurer and averaged to determine the final reported circumference. Waist circumference (WC) was measured at the midway point between the last floating rib and the iliac crest. Neck circumference (NC) was measured with participants standing erect and with their heads positioned in the horizontal Frankfurt plane. The upper edge of the measuring tape was placed just below the laryngeal prominence and applied perpendicularly to the long axis of the neck. Hip circumference was determined at the level of the maximum extension of the buttocks posteriorly in a horizontal plane. Waist-to-hip ratio (WHr) was calculated as WC divided by hip circumference.

Total and percentage body fat mass (FM) and fat-free mass (FFM) were determined by dual-energy X-ray absorptiometry 
(DXA; model 8548 BX1L, Lunar DPX type, software Encore 2005; GE Healthcare Europe GmbH, Freiburg, Germany). Subjects underwent a complete body scan in the supine position. Elderly women were secured by nonelastic straps at the knees and ankles with arms aligned along with the trunk with palms facing the thighs. Appendicular fat-free mass (AFFM) was determined by the sum of the FFM from the lower and upper body.

\section{SO identification}

SO was identified in accordance with specifications proposed in the literature. ${ }^{25}$ Briefly, the method of classification is based on the residual values of a regression equation that predicts AFFM based on height (in meters) and FM (in $\mathrm{kg}$ ). The equation of elderly women has been identified as follows: predicted AFFM $=-14.529+(17.989 \times$ height in meters $)+(0.1307 \times$ total $F M$ in $\mathrm{kg})$. The residual value $(\mathrm{DXA}$ measured AFFM - equation predicted AFFM) is used for the classification of SO, and the cutoff value corresponds to a residual $\leq 3.4$. Obesity was classified by BMI assuming a cutoff point of $27.0 \mathrm{~kg} / \mathrm{m}^{2}{ }^{26}$

\section{Functional tests}

Functional capacity was tested by 30 -second chair stand test, arm curl, and timed "up and go" (TUG) following the recommendations of Jones and Rikli. ${ }^{27}$ During the chair stand up test, subjects were seated and were asked to perform as many squats as possible during 30 seconds. In the arm curl test, the subjects sat on a chair and completed the maximum number of repetitions possible during 30 seconds. The TUG test consisted of standing from a chair and walking in the lowest time possible to a cone $3 \mathrm{~m}$ away, circling around the cone, and returning to the chair.

\section{Muscle strength assessment}

Maximum strength (one repetition maximum [RM]) was tested in chest press, $45^{\circ}$ leg press, and standing arm curl on the same day with 10 minutes rest between exercises. Subjects were allowed to warm-up for 10 minutes with low-intensity treadmill running and performed eight repetitions with an estimated $50 \%$ of 1 RM (according to the loads estimated in the adaptation period). After 1 minute of rest, subjects completed three repetitions with an estimated $70 \%$ of 1 RM and rested for 3 minutes. They had three attempts to reach $1 \mathrm{RM}$ with progressively heavier loads, using 3-5 minutes of rest between trials. Exercise standards followed the recommendations of Brown and Weir. ${ }^{28}$ This procedure was repeated 3 days later to determine test-retest reproducibility. A higher intraclass correlation coefficient was found between the first and the second test days (chest press $r=0.95,45^{\circ}$ leg press $r=0.98$, and arm curl $r=0.98$ ).

\section{RT protocol}

Briefly, subjects underwent 2 weeks of RT familiarization with one exercise for each major muscle group and two sets of 15 submaximal repetitions. This training was based on a previous study from our research group. ${ }^{29}$ Subjects performed two weekly sessions during the 16-week RT. In the first 4 weeks, they completed three sets of 12-14 RM and from week 5 to 8 three sets of 10-12 RM. In weeks 9-12, subjects completed 8-10 RM and from week 13 to 16 three sets of 6-8 RM. All training sessions were supervised by an experienced RT professional. Resistance exercises were performed in the following order: chest press, $45^{\circ}$ leg press, seated low row, leg extension, leg curl, triceps pulley extension, leg adduction and abduction machines, standing arm curl, and seated calf raise. All resistance exercises were completed with three sets leading to concentric failure, and the number of repetitions and rest intervals between sets and exercises followed the proposed periodization. The mean duration to complete one repetition was 3-4 seconds (1-2 seconds for the concentric phase and 2 seconds for the eccentric phase) and training sessions lasted $\sim 40-50$ minutes. Diet was not controlled, though they were advised to maintain similar food ingestion.

\section{Statistical analysis}

Homogeneity of variance was tested using Levene's test. A mixed factor analysis of variance (ANOVA) $2 \times 2$ (groups $\times$ time) was employed to examine differences in the dependent variables. In addition, effect size (ES) of the treatment was calculated according to the following equation previously proposed in the literature: ${ }^{30}$

$$
\text { Prepost ES }=\frac{\text { Posttest mean }- \text { Pretest mean }}{\text { Pretest SD }}
$$

The significance level was set at $P \leq 0.05$. All analyses were performed using the SPSS, version 20.0 (IBM Corporation, Armonk, NY, USA) for Windows. Values are given as mean $\pm \mathrm{SD}$.

\section{Results}

Table 1 presents anthropometric measures and body composition variables at baseline and after the 16-week training period. A significant group $\times$ time interaction was found only for WHr $(\mathrm{F}=5.68, P=0.02)$. Yet, as expected, participants from the SO group presented a significantly higher FM 
Table I Anthropometry and body composition variables at baseline and postintervention

\begin{tabular}{|c|c|c|c|c|}
\hline \multirow[t]{2}{*}{ Variable } & \multicolumn{2}{|l|}{ Baseline } & \multicolumn{2}{|c|}{ Postintervention } \\
\hline & $\begin{array}{l}\text { Non-SO } \\
(n=4 I)\end{array}$ & $\begin{array}{l}\text { SO } \\
(n=8)\end{array}$ & $\begin{array}{l}\text { Non-SO } \\
(n=4 I)\end{array}$ & $\begin{array}{l}\text { SO } \\
(n=8)\end{array}$ \\
\hline FM (kg) & $25.4 \pm 7.2^{\#}$ & $31.7 \pm 9.5$ & $24.8 \pm 7.0^{*, a}$ & $31.7 \pm 9.5$ \\
\hline$\% F M$ & $40.8 \pm 6.0$ & $45.7 \pm 9.0$ & $39.9 \pm 5.6^{*, a}$ & $45.7 \pm 8.1$ \\
\hline $\mathrm{FFM}(\mathrm{kg})$ & $35.7 \pm 4.3$ & $34.9 \pm 2.8$ & $36.0 \pm 4.6$ & $34.8 \pm 3.8$ \\
\hline Body weight (kg) & $63.7 \pm 10.8$ & $69.2 \pm 12.3$ & $63.5 \pm 10.6$ & $68.1 \pm 11.7$ \\
\hline BMI $\left(\mathrm{kg} / \mathrm{m}^{2}\right)$ & $27.8 \pm 4.7$ & $28.4 \pm 5.0$ & $27.9 \pm 4.3$ & $28.0 \pm 4.9$ \\
\hline WC (cm) & $85.1 \pm 11.0$ & $85.8 \pm 13.0$ & $82.8 \pm 9.8 *$ & $84.8 \pm 12.5$ \\
\hline $\mathrm{WHr}$ & $0.86 \pm 0.06$ & $0.82 \pm 0.07$ & $0.84 \pm 0.06^{*}$ & $0.85 \pm 0.08$ \\
\hline $\mathrm{NC}(\mathrm{cm})$ & $34.2 \pm 2.9$ & $33.4 \pm 2.9$ & $33.6 \pm 2.6 *$ & $33.0 \pm 2.7$ \\
\hline
\end{tabular}

Notes: Data expressed as mean \pm SD. *Significant difference versus baseline.

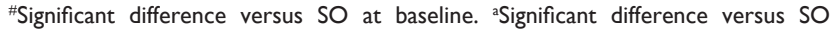
postintervention.

Abbreviations: SO, sarcopenic obesity; FM, fat mass; FFM, fat-free mass; BMI, body mass index; WC, waist circumference; WHr, waist-to-hip ratio; NC, neck circumference.

when compared to non-SO at baseline. This difference did not change after the intervention. Of note, only participants from the non-SO group achieved a significant decrease in FM from baseline to postintervention ( $\mathrm{F}=5.22, P=0.03), \% \mathrm{FM}$ (F=8.44, $P=0.006)$, WC (F=6.80, $P=0.01)$, WHr $(\mathrm{F}=5.86$, $P=0.02)$, and $\mathrm{NC}(\mathrm{F}=5.36, P=0.03)$.

Functional tests did not present significant group $\times$ time interactions. However, Figure $1 \mathrm{~A}$ and $\mathrm{C}$ demonstrates that only the non-SO group improved performance in the 30 -second chair stand test $(\mathrm{F}=29.93, P=0.000)$ and $\mathrm{TUG}$ ( $\mathrm{F}=26.20, P=0.000)$, respectively. On the other hand, both non-SO $(\mathrm{F}=78.95, P=0.000)$ and $\mathrm{SO}(\mathrm{F}=12.51, P=0.001)$ groups presented a better performance in the seated arm curl test after the RT intervention when compared to baseline (Figure 1B). ANOVA showed no significant group $\times$ time interactions regarding $1 \mathrm{RM}$ tests. However, there was a significant time effect for the chest press $(\mathrm{F}=4.21, P=0.05)$, and the non-SO group presented higher strength compared to SO after training for 16 weeks (Figure 2A). In addition, Figure $2 \mathrm{C}$ demonstrates that only the non-SO group showed an increase in the standing arm curl $1 \mathrm{RM}$ when compared to baseline. Alternatively, both non-SO $(\mathrm{F}=79.24, P=0.000)$ and $\mathrm{SO}(\mathrm{F}=6.70, P=0.01)$ groups presented higher leg press strength after the intervention when compared to baseline (Figure 2B).

According to Rhea, ${ }^{30}$ the ES for untrained subjects can be categorized as trivial $(<0.50)$, small $(0.50-1.25)$, moderate $(1.25-1.9)$, and large $(>2.0)$. In general, the present results show that ES ranged from trivial to moderate. Noteworthy, only the non-SO group reached an ES classified as moderate (elbow flexion). Moreover, percent change $(\Delta \%)$ and ES values were higher in non-SO for all variables, with several of the outcomes indicating potentially meaningful differences in the magnitude of effect favoring the non-SO condition (Table 2).

\section{Discussion}

The aim of the present study was to compare the effects of 16 weeks of RT on body composition, muscle strength, and functional capacity of elderly women with and without SO. Our main finding is that important adaptations to RT are attenuated in elderly women with SO and confirm the initial hypothesis. This was evident in anthropometric (Table 1), functional (Figure 1A and C), and strength (Figure 2A and C) parameters. Moreover, the ES of the treatment and percent changes observed between pre- and postintervention (Table 2) corroborate these findings.

Regarding anthropometric data, as opposed to what was observed in the non-SO group, the FM, FFM, WC, and WHr remained virtually unaltered after 12 weeks of RT when compared to pretraining values in SO. These findings are
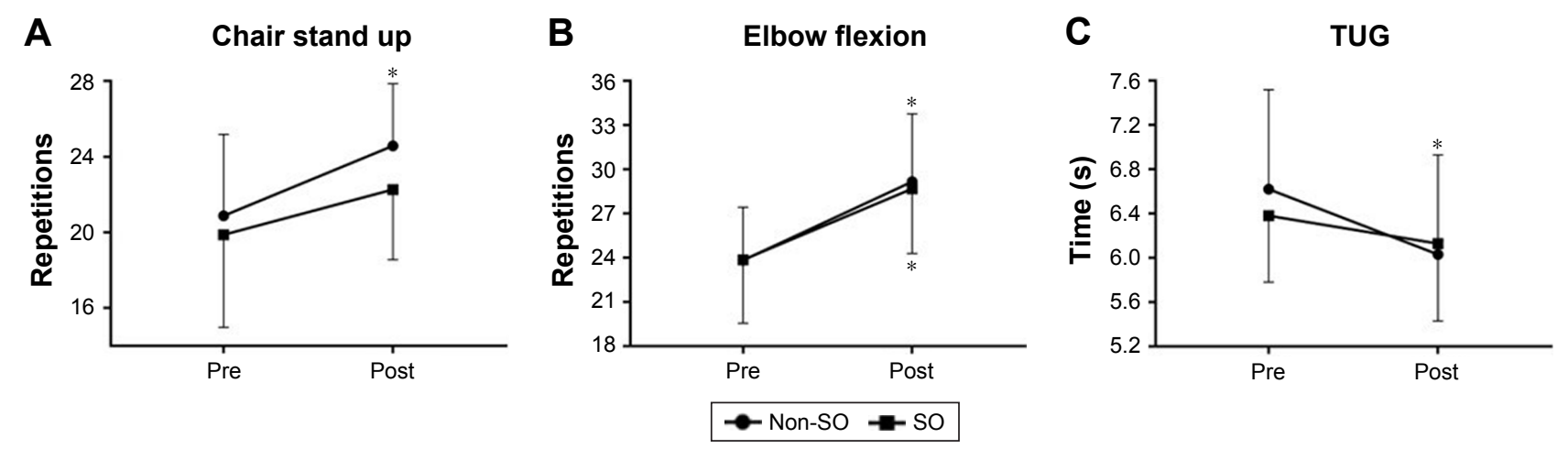

Figure I Functional capacity before and after 16 weeks of RT measured by chair stand up (A), elbow flexion (B), and TUG (C) tests. Note: *Significant difference versus baseline.

Abbreviations: RT, resistance training; SO, sarcopenic obesity; TUG, timed "up and go." 

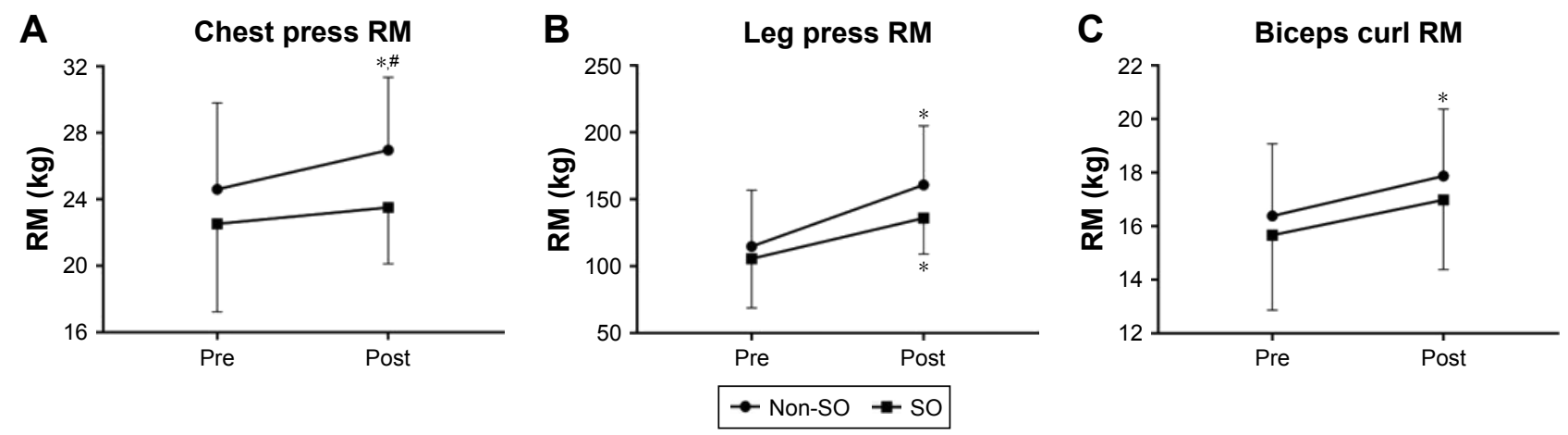

Figure 2 Strength measures before and after 16 weeks of RT measured by chest press RM (A), leg press RM (B), and biceps curl RM (C). Notes: *Significant difference versus baseline. "Significant difference versus SO.

Abbreviations: RT, resistance training; SO, sarcopenic obesity; RM, repetition maximum.

consistent with previous studies where elderly participants with SO trained twice a week for 15 weeks ${ }^{14}$ or three times a week for 24 weeks, ${ }^{31}$ whereby no major effects were noted in adiposity indices, especially when lacking more rigid dietetic control or caloric restriction. ${ }^{14,31}$

Strength assessments have been shown to be more sensitive in predicting balance, mobility, and falls than muscle mass measurements in older obese individuals. ${ }^{32}$ Of interest, gains observed in lower and upper limb extremity strength occur more prominently in non-SO and showed no relation with FFM after our RT protocol. In fact, muscle strength does not always correspond to the amount of $\mathrm{FFM}^{15}$ or muscle mass. ${ }^{11,33}$ Findings of the present study are in line with Balachandran et al, ${ }^{14}$ who reported no major changes in FFM after 15 weeks of RT and employing the same weekly frequency of training (two times per week) utilized in our protocol.

Unlike what was observed in FFM in both groups posttraining, the performance in chair stand up and 30-second chair stand test, as indicators of functional capacity, was improved only in the non-SO group. These results were probably influenced by gains in muscle strength by non-SO

Table 2 Percent change from baseline to postintervention and treatment ES for strength and functional variables

\begin{tabular}{|c|c|c|c|c|}
\hline \multirow[t]{2}{*}{ Variable } & \multicolumn{2}{|c|}{ Non-SO $(n=4 I)$} & \multicolumn{2}{|l|}{ SO $(n=8)$} \\
\hline & $\Delta \%$ & ES & $\Delta \%$ & ES \\
\hline RM leg press (kg) & $50.3 \pm 44.6$ & 1.10 & $40.5 \pm 46.6$ & 0.82 \\
\hline RM chest press $(\mathrm{kg})$ & $12.9 \pm 22.8$ & 0.45 & $8.7 \pm 23.5$ & 0.19 \\
\hline RM biceps curl (kg) & $11.3 \pm 19.8$ & 0.54 & $10.3 \pm 19.4$ & 0.47 \\
\hline Elbow flexion (reps) & $23.8 \pm 20.3$ & 1.48 & $21.4 \pm 12.2$ & 1.10 \\
\hline Sit-to-stand (reps) & $21.4 \pm 25.3$ & 0.85 & $14.8 \pm 16.9$ & 0.48 \\
\hline TUG (s) & $-8.4 \pm I I .5$ & 0.60 & $-3.9 \pm 6.6$ & 0.38 \\
\hline
\end{tabular}

Note: ES: trivial $(<0.50)$, small $(0.50-1.25)$, moderate $(1.25-1.9)$, and large $(>2.0)$. Abbreviations: RM, repetition maximum; TUG, timed "up and go"; SO, sarcopenic obesity; ES, effect size. subjects, as observed in biceps curl and chest press, which did not occur in SO, or were attenuated, as demonstrated in leg press strength in $\mathrm{SO}$, suggesting that adaptations to RT are mitigated in elderly women with SO. This effect could be partially explained by lipotoxicity induced by an increase of intramyocellular triacylglycerol content and fatty acids derived as ceramides and diacylglycerol, ${ }^{34}$ which perpetuate inflammation and impaired single-fiber contractility. ${ }^{35}$ Muscle fat accumulation may also interfere with signaling pathways involved in muscle response to anabolic stimuli, blunting the activation of protein synthesis. ${ }^{19,20}$ In support of this hypothesis, Delmonico et $\mathrm{al}^{33}$ reported that the loss of knee extensor strength with aging coincides with a gain in muscle fat infiltration in men and women with $\mathrm{SO}$, suggesting a pivotal role of fatty acids and derivatives in lowering muscle strength and power, and a lower response to RT in $\mathrm{SO}$, as reported in the present work.

Our results corroborate the findings of Vasconcelos et al, ${ }^{11}$ who reported that 14 older women (aged 65-80 years) with SO showed no improvements in knee extensor isokinetic muscle strength after 15 weeks of RT compared to baseline and to a control group that did not engage in RT (also composed of sarcopenic obese participants). This is of particular importance since women with SO displayed a 2.60 higher odds of having difficulty climbing stairs and a 2.35 higher odds of having difficulty descending stairs, ${ }^{36}$ suggesting a decline in the ability to perform activities of daily living. It is interesting to highlight that in our intervention as well as in the study of Vasconcelos et al, ${ }^{11}$ both of which found a lack of response for improving the physical function of older women with SO, the individuals trained twice per week, while RT protocols employing three sessions per week totaling $30^{28}$ or 24 weeks $^{31}$ improved physical function. This suggests that elderly people with SO may require a higher weekly 
frequency of exercise to significantly impact training-induced increases in muscle strength and functional capacity.

Since gains in muscle strength and functional capacity, as well as a reduction in adiposity, were attenuated in the SO group in our study, it is possible that elderly women with SO may need a longer term of RT intervention for responses to be more perceptible. In addition to the need for higher training volumes as aforementioned, lifestyle interventions with diet-induced adiposity loss and exercise as well as aerobic training inclusion may be required as well. Similarly, other studies have demonstrated that interventions including dietary changes and a combination of aerobic exercise and strength training were more effective than diet or exercise alone in eliciting improvements in physical function and adiposity indices. ${ }^{15-17,37}$

The present study has some limitations that are worthy of note. First, blood samples for SO-related biological and clinical markers were not analyzed. These assessments could have shed mechanistic light on the results. Second, the sample size was different between groups and substantially smaller within the SO group due to time- and cost-related aspects related to DXA testing. However, participants were limited to women, thereby removing the potential influence of gender. Thus, the present study provides unique and novel insights regarding SO adaptations to regimented RT. Moreover, we used a definition of SO previously proposed ${ }^{38}$ and validated ${ }^{25}$ in the literature, helping to ensure credibility of our findings.

\section{Conclusion}

In summary, data from the present investigation show that RT adaptations are attenuated in elderly women with SO compared with those without SO. This indicates that people living with SO may need more time to respond to RT. Of note, the training frequency of the present intervention was only two times a week. Future studies should further investigate chronic effects of RT on SO in larger samples and different frequencies of training.

\section{Disclosure}

The authors report no conflicts of interest in this work.

\section{References}

1. Yang L, Colditz GA. Prevalence of overweight and obesity in the United States, 2007-2012. JAMA Intern Med. 2015;175(8):1412-1413.

2. Flegal KM, Kruszon-Moran D, Carroll MD, Fryar CD, Ogden CL. Trends in obesity among adults in the United States, 2005 to 2014. JAMA. 2016;315(21):2284-2291.

3. Cruz-Jentoft AJ, Baeyens JP, Bauer JM, et al. Sarcopenia: European consensus on definition and diagnosis: report of the European Working Group on Sarcopenia in Older People. Age Ageing. 2010;39(4): 412-423.
4. Roubenoff R. Sarcopenic obesity: does muscle loss cause fat gain? Lessons from rheumatoid arthritis and osteoarthritis. Ann N Y Acad Sci. 2000;904:553-557.

5. Kyle UG, Pirlich M, Lochs H, Schuetz T, Pichard C. Increased length of hospital stay in underweight and overweight patients at hospital admission: a controlled population study. Clin Nutr. 2005;24(1): 133-142.

6. Jarosz PA, Bellar A. Sarcopenic obesity: an emerging cause of frailty in older adults. Geriatr Nurs. 2009;30(1):64-70.

7. Atkins JL, Whincup PH, Morris RW, Lennon LT, Papacosta O, Wannamethee SG. Sarcopenic obesity and risk of cardiovascular disease and mortality: a population-based cohort study of older men. J Am Geriatr Soc. 2014;62(2):253-260.

8. Kim TN, Choi KM. The implications of sarcopenia and sarcopenic obesity on cardiometabolic disease. J Cell Biochem. 2015;116(7): 1171-1178.

9. Baumgartner RN, Wayne SJ, Waters DL, Janssen I, Gallagher D, Morley JE. Sarcopenic obesity predicts instrumental activities of daily living disability in the elderly. Obes Res. 2004;12(12):1995-2004.

10. Cesari M, Rolland Y, Abellan Van Kan G, Bandinelli S, Vellas B, Ferrucci L. Sarcopenia-related parameters and incident disability in older persons: results from the "invecchiare in Chianti" study. J Gerontol A Biol Sci Med Sci. 2015;70(4):457-463.

11. Vasconcelos KS, Dias JM, Araújo MC, Pinheiro AC, Moreira BS, Dias RC. Effects of a progressive resistance exercise program with highspeed component on the physical function of older women with sarcopenic obesity: a randomized controlled trial. Braz J Phys Ther. 2016; 20(5):432-440.

12. Huang SW, Ku JW, Lin LF, Liao CD, Chou LC, Liou TH. Body composition influenced by progressive elastic band resistance exercise of sarcopenic obesity elderly women: a pilot randomized controlled trial. Eur J Phys Rehabil Med. 2017;53(4):556-563.

13. Chen HT, Chung YC, Chen YJ, Ho SY, Wu HJ. Effects of different types of exercise on body composition, muscle strength, and IGF-1 in the elderly with sarcopenic obesity. J Am Geriatr Soc. 2017;65(4): 827-832.

14. Balachandran A, Krawczyk SN, Potiaumpai M, Signorile JF. Highspeed circuit training vs hypertrophy training to improve physical function in sarcopenic obese adults: a randomized controlled trial. Exp Gerontol. 2014;60:64-71.

15. Villareal DT, Banks M, Sinacore DR, Siener C, Klein S. Effect of weight loss and exercise on frailty in obese older adults. Arch Intern Med. 2006;166(8):860-866.

16. Frimel TN, Sinacore DR, Villareal DT. Exercise attenuates the weightloss-induced reduction in muscle mass in frail obese older adults. Med Sci Sports Exerc. 2008;40(7):1213-1219.

17. Villareal DT, Chode S, Parimi N, et al. Weight loss, exercise, or both and physical function in obese older adults. N Engl J Med. 2011; 364(13):1218-1229.

18. Prestes J, Shiguemoto G, Botero JP, et al. Effects of resistance training on resistin, leptin, cytokines, and muscle force in elderly postmenopausal women. J Sports Sci. 2009;27(14):1607-1615.

19. Guillet C, Delcourt I, Rance M, et al. Changes in basal and insulin and amino acid response of whole body and skeletal muscle proteins in obese men. J Clin Endocrinol Metab. 2009;94(8):3044-3050.

20. Tardif N, Salles J, Guillet C, et al. Muscle ectopic fat deposition contributes to anabolic resistance in obese sarcopenic old rats through eIF2 $\alpha$ activation. Aging Cell. 2014;13(6):1001-1011.

21. Hamrick MW, McGee-Lawrence ME, Frechette DM. Fatty infiltration of skeletal muscle: mechanisms and comparisons with bone marrow adiposity. Front Endocrinol (Lausanne). 2016;7:69.

22. Nilsson MI, Dobson JP, Greene NP, et al. Abnormal protein turnover and anabolic resistance to exercise in sarcopenic obesity. FASEB J. 2013;27(10):3905-3916.

23. Rivas DA, Morris EP, Haran PH, et al. Increased ceramide content and $\mathrm{NF} \kappa \mathrm{B}$ signaling may contribute to the attenuation of anabolic signaling after resistance exercise in aged males. J Appl Physiol. 2012;113(11): $1727-1736$. 
24. US Department of Health and Human Services; National Institute of Diabetes and Digestive and Kidney Diseases. Understanding Adult Overweight and Obesity. Available from: https://www.niddk.nih.gov/ health-information/weight-management/adult-overweight-obesity//. Accessed March 2, 2018.

25. Oliveira RJ, Bottaro M, Júnior JT, Farinatti PTV, Bezerra LA, Lima RM. Identification of sarcopenic obesity in postmenopausal women: a cutoff proposal. Braz J Med Biol Res. 2011;44(11):1171-1176.

26. Lipschitz DA. Screening for nutritional status in the elderly. Prim Care. 1994;21(1):55-67.

27. Rikli RE, Jones CJ. Development and validation of a functional fitness test for community residing older adults. J Aging Phys Act. 1999; 7(2):129-161.

28. Brown LE, Weir JP. ASEP procedures recommendation I: accurate assessment of muscular strength and power. J Exerc Physiol. 2001; 4(3):1-21.

29. Prestes J, Frollini AB, de Lima C, et al. Comparison between linear and daily undulating periodized resistance training to increase strength. J Strength Cond Res. 2009;23(9):2437-2442.

30. Rhea MR. Determining the magnitude of treatment effects in strength training research through the use of the effect size. J Strength Cond Res. 2004;18(4):918-920.

31. Gadelha AB, Paiva FM, Gauche R, de Oliveira RJ, Lima RM. Effects of resistance training on sarcopenic obesity index in older women: a randomized controlled trial. Arch Gerontol Geriatr. 2016;65:168-173.
32. Menant JC, Weber F, Lo J, et al. Strength measures are better than muscle mass measures in predicting health-related outcomes in older people: time to abandon the term sarcopenia? Osteoporos Int. 2017; 28(1):59-70.

33. Delmonico MJ, Harris TB, Visser M, et al. Longitudinal study of muscle strength, quality, and adipose tissue infiltration. Am J Clin Nutr. 2009;90(6):1579-1585.

34. Kitessa SM, Abeywardena MY. Lipid-induced insulin resistance in skeletal muscle: the chase for the culprit goes from total intramuscular fat to lipid intermediates, and finally to species of lipid intermediates. Nutrients. 2016;8(8):pii:E466.

35. Tumova J, Andel M, Trnka J. Excess of free fatty acids as a cause of metabolic dysfunction in skeletal muscle. Physiol Res. 2016;65(2): 193-207.

36. Rolland Y, Lauwers-Cances V, Cristini C, et al. Difficulties with physical function associated with obesity, sarcopenia, and sarcopenic-obesity in community-dwelling elderly women: the EPIDOS (EPIDemiologie de 1'OSteoporose) Study. Am J Clin Nutr. 2009;89(6):1895-1900.

37. Prior SJ, Joseph LJ, Brandauer J, Katzel LI, Hagberg JM, Ryan AS. Reduction in midthigh low-density muscle with aerobic exercise training and weight loss impacts glucose tolerance in older men. $J$ Clin Endocrinol Metab. 2007;92(3):880-886.

38. Newman AB, Kupelian V, Visser M, et al. Sarcopenia: alternative definitions and associations with lower extremity function. J Am Geriatr Soc. 2003;51(11):1602-1609.
Clinical Interventions in Aging

\section{Publish your work in this journal}

Clinical Interventions in Aging is an international, peer-reviewed journal focusing on evidence-based reports on the value or lack thereof of treatments intended to prevent or delay the onset of maladaptive correlates of aging in human beings. This journal is indexed on PubMed Central, MedLine,

\section{Dovepress}

CAS, Scopus and the Elsevier Bibliographic databases. The manuscript management system is completely online and includes a very quick and fair peer-review system, which is all easy to use. Visit http://www.dovepress. com/testimonials.php to read real quotes from published authors. 